\title{
Hereditary breast cancer: molecular biology and management update
}

\author{
Hiroko Yamashita ${ }^{1}$
}

Received: 14 August 2017 / Accepted: 16 August 2017 / Published online: 24 August 2017

(C) Japan Society of Clinical Oncology 2017

\section{Introduction}

For $B R C A 1$ and $B R C A 2$ carriers, lifetime estimates of breast cancer risk range from $49 \%$ to $88 \%$, whereas the risk of ovarian cancer ranges from $40 \%$ to $59 \%$, and from $18 \%$ to $35 \%$, for $B R C A 1$ or $B R C A 2$ carriers, respectively [1, 2]. Approximately $5 \%$ of unselected patients with breast cancer carry a germline BRCA mutation. The identification of $B R C A l$ and $B R C A 2$ mutations permits the implementation of prevention strategies, including screening by magnetic resonance imaging or risk-reducing surgeries. As tumor suppressor genes, $B R C A 1$ and $B R C A 2$ encode proteins involved in the repair of DNA double-strand breaks by way of a homologous recombination repair pathway. Members of the poly (ADP-ribose) polymerase (PARP) family of enzymes are central to the repair of DNA single-strand breaks. Consequently, the oral PARP inhibitor, olaparib, is approved for the treatment of patients with recurrent ovarian cancer and a BRCA mutation. Moreover, olaparib has also been shown to have significant benefits compared with standard therapy in patients with metastatic breast cancer and a germline $B R C A$ mutation [3]. In addition, since the initial discovery that pathogenic germline alterations in $B R C A 1$ and $B R C A 2$ genes increase susceptibility to breast and ovarian cancers, many genes have subsequently been discovered that also increase breast cancer risk [4]. Advances in technology have resulted in the ability to test for multiple genes associated with a hereditary predisposition to breast cancer.

Hiroko Yamashita

hirokoy@huhp.hokudai.ac.jp

1 Department of Breast Surgery, Hokkaido University Hospital, Kita 14 Nishi 5, Kita-ku, Sapporo 060-8648, Japan
The invited review articles present an overview of the current status of the molecular biology and management of hereditary breast cancer. In the first invited article, Professor Yoshio Miki, who cloned the BRCAl gene in 1994 [5], highlights basic aspects of the function and deficiencies of the BRCAl gene. In the second invited article, Dr. Hideko Yamauchi describes the management of hereditary breast cancer and discusses important issues in caring for patients and families in Japan with a BRCA mutation.

Compliance with ethical standards

Conflict of interest The author has no conflict of interest.

\section{References}

1. Chen S, Parmigiani G (2007) Meta-analysis of BRCA1 and BRCA2 penetrance. J Clin Oncol 25:1329-1333

2. van der Kolk DM, de Bock GH, Leegte BK et al (2010) Penetrance of breast cancer, ovarian cancer and contralateral breast cancer in BRCA1 and BRCA2 families: high cancer incidence at older age. Breast Cancer Res Treat 124:643-651

3. Robson M, Im SA, Senkus E et al (2017) Olaparib for metastatic breast cancer in patients with a germline BRCA mutation. N Engl J Med 377:523-533

4. Nielsen FC, van Overeem Hansen T, Sorensen CS (2016) Hereditary breast and ovarian cancer: new genes in confined pathways. Nat Rev Cancer 16:599-612

5. Miki Y, Swensen J, Shattuck-Eidens D et al (1994) A strong candidate for the breast and ovarian cancer susceptibility gene BRCA1. Science 266:66-71 